\title{
Contaminación con huevos de Toxocara spp en parques públicos del distrito de La Molina, Lima, y su relación con el programa de vigilancia sanitaria de parques y jardines
}

\author{
Contamination with eggs of Toxocara spp in public parks of La Molina district, \\ Lima, and its relationship with the sanitary surveillance programme \\ of parks and gardens
}

\author{
Carlos Malca V. ${ }^{1}$, Amanda Chávez V., ${ }^{1,2}$, Rosa Pinedo V. ${ }^{1}$, Deisy Abad-Ameri ${ }^{1}$
}

\section{Resumien}

\begin{abstract}
El objetivo del estudio fue determinar la contaminación de los parques públicos del distrito de La Molina, Lima, con huevos de Toxocara spp y su relación con la categorización de los parques (no amigables, amigables y poco amigables) dentro del Programa de Vigilancia Sanitaria de Parques y Jardines, elaborado por la Dirección de Higiene Alimentaria y Zoonosis de la DIGESA - Ministerio de Salud (MINSA). El estudio fue de tipo descriptivo, evaluándose 131 parques públicos entre agosto de 2014 y abril de 2016. Muestras representativas de tierra y césped $(1-2 \mathrm{~kg})$ por parque fueron colectadas mediante la técnica de la doble $\mathrm{W}$ y analizadas mediante las técnicas de sedimentación y flotación con solución sobresaturada de $\mathrm{NaCl}$. Se consideró como parque positivo la presencia de al menos un huevo de Toxocara spp en la muestra. La categorización de los parques fue realizada por personal de La Municipalidad de La Molina, con base a tres criterios (infraestructura adecuada, ambiente y riesgos sanitarios), según la «Ficha de Evaluación Sanitaria Ambiental de Parques y Jardines» del DIGESA-MINSA. Solo un parque resultó positivo a huevos de Toxocara spp, representando una prevalencia de $0.76 \%$. Paralelamente, cinco parques fueron considerados como no amigables, 75 como amigables y 51 como poco amigables; perteneciendo a este último grupo el parque positivo. No se pudo relacionar ambos resultados por el escaso número de parques positivos. El bajo porcentaje de parques contaminados se debe probablemente a la implementación por el municipio del Programa de Vigilancia Sanitaria de Parques y Jardines (tenencia responsable, recojo de excretas, etc.), así como de las constantes mejoras realizadas desde el 2011 y por las frecuentes campañas de desparasitación y charlas informativas a los dueños de las mascotas.
\end{abstract}

Palabras clave: Toxocara spp; toxocariasis; zoonosis; parques públicos

\footnotetext{
${ }^{1}$ Laboratorio de Microbiología y Parasitología Veterinaria, Facultad de Medicina Veterinaria, Universidad Nacional Mayor de San Marcos, Lima, Perú

${ }^{2}$ E-mail: achavezvg@gmail.com
}

Recibido: 8 de septiembre de 2018

Aceptado para publicación: 11 de febrero de 2019 
The aim of this study was to determine the contamination of public parks of La Molina district in Lima with eggs of Toxocara spp and its relationship with the categorization of the parks (unfriendly, friendly and not very friendly) within the Health Surveillance Programme of Parks and Gardens, prepared by the Department of Food Hygiene and Zoonoses of DIGESA - Ministry of Health (MINSA). The study was of descriptive type, evaluating 131 public parks between August 2014 and April 2016. Representative samples of soil and grass (1-2 kg) per park were collected using the double $\mathrm{W}$ technique and analysed by the techniques of sedimentation and flotation with supersaturated $\mathrm{NaCl}$ solution. The presence of at least one Toxocara spp egg in the sample was considered positive. The categorization of the parks was carried out by La Molina Municipality staff, based on three criteria (adequate infrastructure, environment and sanitary risks) according to the «Environmental Sanitary Assessment Card for Parks and Gardens» of DIGESA-MINSA. Only one park was positive to eggs of Toxocara spp, representing a prevalence of $0.76 \%$. In parallel, five parks were considered unfriendly, 75 as friendly and 51 as not very friendly, where the positive park was a not very friendly one. It was not possible to relate both results due to the small number of positive parks. The low percentage of contaminated parks was probably due to the implementation by the municipality of the Sanitary Vigilance Programme of Parks and Gardens (responsible tenure, excreta collection, etc.), as well as the constant improvements made since 2011 and the frequent deworming campaigns and informative talks to pet owners.

Key words: Toxocara spp; toxocariasis; zoonoses; public parks

\section{INTRODUCCIÓN}

La toxocariosis es una enfermedad zoonótica de origen parasitario de importancia en la salud pública. La infección humana es accidental por la ingestión de huevos con larvas infectivas de nematodos del género Toxocara; siendo $T$. canis y $T$. cati, parásitos intestinales de perros y gatos, respectivamente, los dos más importantes para el ser humano (Huapaya et al., 2009). Perros y gatos infectados expulsan en sus heces los huevos del parásito contaminando los parques públicos y áreas verdes (Alonso et al., 2006).

Los huevos de estos parásitos pueden sobrevivir en condiciones adecuadas hasta tres años en el suelo, lo que eleva las posibilidades de infección (Huapaya et al., 2009; Armstrong et al., 2011). La infección humana es accidental, principalmente por ingestión de los huevos al estar en contacto con áreas de tierra contaminadas con huevos del parásito (Archelli y Kozubsky, 2008; Huapaya et al., 2009), así como por el consumo de vegetales contaminados (Omodu et al., 2003), carne poco cocida procedente de hospederos paraténicos que contienen larvas encapsuladas (Zibaei et al., 2010), por contacto directo con el pelaje de perros infectados (Romero et al., 2009), y por onicofagia y manos mal lavadas (Canese et al., 2003; Breña et al., 2011). La ingestión de estos huevos infectivos por el hombre (especialmente niños) ocasiona enfermedades conocidas como Síndrome de la Larva Migrante Visceral (LMV) y Síndrome de la Larva Migrante Ocular (LMO) (Schanstz y Glickman, 1981; Acha y Szyfres, 2003).

Se han realizado diversos estudios para determinar el grado de contaminación con huevos de Toxocara spp en los parques públicos de diversos países de América. Así, se reportó 56.5\% en La Plata, Argentina (Cór- 
doba et al., 2002) y de 69.2 y $95.4 \%$ en verano e invierno, respectivamente, en Maringá, Brasil (Tiyo et al., 2008), en tanto que Canese et al. (2003) reportaron una contaminación de $53 \%$ en arenas de plazas y parques de Asunción, Paraguay.

Estudios anteriores realizados en el departamento de Lima y otras provincias del Perú revelan diversas prevalencias de huevos de Toxocara spp en suelos de parques y áreas recreativas (Velarde et al., 1999; Cajas et al., 2000; Serrano et al., 2000; La Rosa et al., 2001; Chávez et al., 2002). Se dispone de reportes desde $20.7 \%$ en perros y $14.3 \%$ en gatos (Noé et al., 2011) en el cono norte de Lima hasta 63.4\% en Lima Oeste (López et al., 2005). A raíz de estos resultados, el Ministerio de Salud, a través de La Dirección de Higiene Alimentaria y Zoonosis (DHAZ), órgano de línea de la Dirección General de Salud Ambiental (DIGESA) viene trabajando desde el año 2009 con el «Programa de Vigilancia Sanitaria de Parques y Jardines» en las DISAS/DIRESAS de Lima y Callao, con el fin de evaluar las condiciones sanitarias y ambientales de los parques a fin de proteger la salud pública. El programa busca minimizar factores de riesgo como presencia de canes que defecan en parques, suelos contaminados con huevos de Toxocara $\mathrm{sp}$, presencia de roedores, etc. (DIGESA, 2009). La acción conjunta entre DIGESA y las municipalidades basadas en capacitación del personal, diagnostico sanitario ambiental de los parques, campañas de desparasitación gratuitas, instalación de letreros de tenencia responsable y recojo de excretas y charlas informativas de concientización hacia los dueños de las mascotas ameritan una reevaluación de los parques públicos para actualizar y evaluar la dinámica de diseminación de esta enfermedad.

El objetivo del estudio fue determinar la contaminación de los parques públicos del distrito de La Molina, Lima, con huevos de Toxocara spp, relacionando sus resultados con la categorización de los parques (no amigables, amigables y poco amigables) dentro del Programa de Vigilancia Sanitaria de Parques y Jardines, elaborado por la Dirección de Higiene Alimentaria y Zoonosis de la DIGESA-Ministerio de Salud (MINSA).

\section{Materiales y Métodos}

\section{Lugar de Estudio}

El estudio se realizó en los parques públicos del distrito de La Molina, Lima, Perú, entre agosto de 2014 y abril de 2016, el cual se encuentra ubicado a una altitud de 350$900 \mathrm{msnm}$, con clima, por lo general, templado $\left(13-31{ }^{\circ} \mathrm{C}\right)$. Tiene una superficie de 65.75 $\mathrm{km}^{2}$, de los cuales $5.4 \mathrm{~km}^{2}$ son áreas verdes. Así mismo, cuenta con 190 parques debidamente implementados (Munimolina, 2016). Siguiendo las recomendaciones del área sanitaria del DIGESA, dichos parques públicos son regados dos veces por semana. Cuentan con personal de limpieza y mantenimiento, quienes a su vez, están encargados de la eliminación de basura y recojo de excretas (Munimolina, 2016).

\section{Tamaño Muestral}

El tamaño de la muestra se calculó por medio de la fórmula de poblaciones finitas (Daniel, 1996). Se empleó un nivel de confianza del $95 \%$, un error del 5\%, una prevalencia esperada del $55.9 \%$ (Serrano et al., 2000) y 190 parques en total. El tamaño muestral calculado fue de 127 parques; sin embargo, en el presente estudio, se analizaron 131 parques públicos bajo el sistema de muestreo aleatorio simple (Daniel, 1996).

\section{Toma de Muestra}

Las muestras de césped (pasto y tierra), se colectaron según el método de la doble W. Se trazó imaginariamente dos W en dirección opuesta entre sí, en el área total del parque a muestrear. Luego, se contabilizó el número total de pasos en cada una de las $\mathrm{W}$ y se obtuvo el número correspondiente al 10\% 
de pasos de cada W. Las muestras se tomaron, luego de caminar el número de pasos obtenidos previamente. Desde cada posición, se colectaron 20-50 g de césped con la ayuda de un cuchillo o pala con una profundidad no mayor de $2 \mathrm{~cm}$ de diámetro (Guerrero, 1975). Todas las muestras de césped de ambas W fueron colectadas dentro de una misma bolsa plástica, obteniéndose una única muestra por parque. Así mismo, la cantidad de césped obtenido por parque dependió de su extensión, llegándose a colectar alrededor entre 1 y $2 \mathrm{~kg}$ por parque.

\section{Procesamiento y Análisis}

Las muestras fueron colocadas en bolsas plásticas y trasladadas de inmediato al Laboratorio de Parasitología de la Facultad de Medicina Veterinaria (FMV) de la Universidad Nacional Mayor de San Marcos (UNMSM) para ser procesadas mediante las técnicas de sedimentación y flotación con solución sobresaturada de sal. Para esto, la muestra de tierra y césped de cada parque fue homogenizada, se vertió $1 \mathrm{~kg}$ en un balde con 10 litros de agua, dejándolo reposar por no menos de 11 horas o durante toda la noche. Posteriormente, el contenido fue filtrado a través de dos tamices ( 8 y 60 hilos por pulgada) y luego dos veces más utilizando solo el tamiz de 60 hilos por pulgada. Se dejó sedimentar por 2 horas.

Se descartó el sobrenadante y se resuspendió el sedimento en un balde de $3 \mathrm{~L}$ con solución sobresaturada de sal ( $360 \mathrm{~g}$ de sal por litro), lo cual permite la flotación de los huevos. Luego de 15 minutos de procedió a recolectar los huevos de la superficie con una placa Petri. Esta operación se repitió varias veces lavando la placa Petri y recepcionando el lavado en una copa de precipitación $(500 \mathrm{ml})$. Por último, se dejó reposar por 1-2 horas y se descartó el sobrenadante. Con una pipeta Pasteur se evaluó todo el sedimento, colocando 1-2 gotas en una lámina portaobjeto, la cual se observó al microscopio. La muestra fue considerada positiva con la presencia de uno o más huevos de Toxocara spp.

\section{Clasificación de los Parques Públicos}

En el «Programa de Vigilancia Sanitaria de Parques y Jardines» de la Dirección de Higiene Alimentaria y Zoonosis (DHAZ) del Ministerio de Salud, los parques públicos han sido categorizados con base a la «Ficha de Evaluación Sanitaria Ambiental de Parques y Jardines» elaborada por DIGESA, la cual se centra en el cumplimiento de tres criterios de evaluación: «infraestructura adecuada» (presencia de iluminación pública, veredas, senderos, etc.), «ambiente» (ausencia de residuos sólidos y montículos de maleza, presencia de depósitos para deposiciones de canes, etc.) y «riesgos sanitarios» (suministro de agua potable, ausencia de excretas humanas y caninas, presencia de depósitos de basura con bolsas, ausencia de madrigueras de roedores, etc.).

El puntaje máximo es de 84 puntos por parque, el cual representa el $100 \%$ del porcentaje de cumplimiento. Los parques se clasifican en tres tipos: Parques Amigables (6584 puntos), poco amigables (43-64 puntos) y no amigables ( $0-42$ puntos), entendiéndose como parques amigables a todos aquellos en donde se han logrado minimizar los factores de riesgo para la salud de la población (DIGESA, 2009).

\section{Análisis de la Información}

Se calculó la prevalencia de Toxocara spp mediante la determinación del número de parques públicos positivos. La prevalencia fue expresada en forma porcentual con sus respectivos intervalos de confianza al 95\% (Daniel, 1996). No obstante, no se pudo evaluar la posible asociación entre la variable clasificación del parque con la presencia del parásito debido al escaso número de parques positivos encontrados.

\section{Consideraciones Éticas}

Todos los procedimientos del presente estudio contemplaron los lineamientos de buenas prácticas y ética en investigación 
biomédica siguiendo las normas reglamentadas por el Comité de Ética de la FMVUNMSM.

\section{Resultados y Discusión}

La prevalencia de Toxocara spp en parques públicos del distrito de La Molina fue 0.76\% (Cuadro 1). De los 131 parques evaluados, solo se halló un parque positivo a la presencia de huevos de Toxocara spp. En la categorización de los parques públicos realizada por personal de La Municipalidad de La Molina se determinó que cinco parques públicos fueron considerados como no amigables, 51 poco amigables y 75 amigables. El parque positivo estuvo considerado como parque poco amigable.

La baja prevalencia hallada en el presente estudio difiere con diversos reportes realizados en la región de Lima y provincias. Así, Dávalos et al. (2000) reportaron 52.5\% en Chincha Alta, Ica; Montalvo et al. (2016) encontraron el $100 \%$ de parques positivos en el distrito de Amarilis, región Huánuco, Aguinaga et al. (2002) hallaron $100 \%$ de positividad en Ferreñafe, y Goicochea (2012) encontró el $52.1 \%$ en el distrito de Trujillo, La Libertad, mientras que Cáceres et al. (2017) reportaron una prevalencia de $66.7 \%$ en la ciudad de Abancay. Por otro lado, en Lima se determinó $37 \%$ de parques positivos en la Provincia Constitucional del Callao (Velarde, 1999), 29.6\% en el Cono Sur (Cajas et al., 2000), 34,3\% en el Cono Norte (La Rosa et al., 2001), 63\% en el Cono Oeste (López et al., 2005) y $41.1 \%$ en el Cono Este de Lima (Serrano et al., 2000), estudio donde se reporta un 55.9\% (19/34) en el distrito de La Molina.

Si bien solo en 1 de los 131 parques analizados pudo observarse la presencia de huevos de Toxocara spp mediante la técnica de sedimentación y flotación, esto no indica necesariamente que en los otros parques públicos evaluados no haya huevos de este pará-
Cuadro 1. Prevalencia de Toxocara spp en parques públicos del distrito de La Molina, Lima, Perú

\begin{tabular}{lccc}
\hline \multirow{2}{*}{ Categorías } & \multirow{2}{*}{ Total } & \multicolumn{2}{c}{ Positivos } \\
\cline { 3 - 4 } & & $\mathrm{n}$ & $\%$ \\
\hline No amigable & 5 & 0 & 0 \\
Poco amigable & 51 & 1 & 1.96 \\
Amigable & 75 & 0 & 0 \\
\hline Total $(\% \pm$ IC) & 131 & 1 & 0.76 \\
\hline
\end{tabular}

sito, sino que la cantidad relativa de huevos se encontraría por debajo del límite de detección de los métodos utilizados, lo cual sugiere que el grado de contaminación sería mínima (Canese et al., 2003).

Así mismo, cabe mencionar, que los diversos estudios sobre la contaminación de parques realizados en los distritos de Lima entre los años 1999 y 2001, evidenciaron que entre 30 y $63 \%$ presentaban huevos de Toxocara spp, donde la clasificación de los parques fue realizada según su estado de conservación como parques bien conservados (césped en toda su área), medianamente conservados (césped en cerca del 50\% del área) y mal conservados (sin césped) o baldíos (Velarde, 1999; Cajas et al., 2000; Serrano et al., 2000; La Rosa et al., 2001; Chávez et al., 2002), dichos resultados no reflejan la situación sanitaria actual de los parques y jardines públicos de la ciudad de Lima.

La clasificación anterior de parques públicos, donde los parques bien y medianamente conservados eran los más contaminados con huevos de Toxocara spp, dado que presentaban áreas con vegetación y tierras húmedas, umbrosas, coloidales y compactas proporcionando condiciones como humedad y sombra que favorecían el desarrollo de los huevos de parásitos, entre ellos los de Toxocara spp. Por otro lado, en los parques 
mal conservados con terrenos secos, blandos y arenosos, los huevos estarían expuestos a la desecación y a la acción directa de los rayos solares que en corto tiempo los destruían (Serrano et al., 2000; Chávez et al., 2002; López et al., 2005). Sin embargo, en este tipo de clasificación no se evaluaban otros factores como presencia de depósitos de basura, tenencia responsable y recojo de excretas por parte de los dueños de las mascotas y compromiso de la municipalidad con la mejora e implementación de los parques públicos.

DIGESA (2009) reportó hace nueve años los primeros resultados del «Programa Piloto», realizados en 86 parques de diferentes direcciones de salud (DISAS) y direcciones regionales de salud (DIRESAS) de Lima y Callao, indicando que el $33.7 \%$ de parques estaban contaminados con huevos de Toxocara spp, habiendo la mayor concentración de parques contaminados en la DISA IV Lima-Este (46.7\%), que es donde se encuentra el distrito de La Molina. En esa primera evaluación de 86 parques en La Molina, 34 resultaron ser parques no amigables, 30 poco amigables y solo 22 amigables; resultados muy diferentes a los hallados en el presente estudio, dado que en aquella época no estaban instauradas todas las mejoras sanitarias y de control dictaminadas por DIGESA que cuentan hoy en día los parques.

Es probable que el bajo porcentaje de parques contaminados hallado en el presente estudio $(0.76 \%)$ se deba a las mejoras realizadas por la Municipalidad del Distrito de La Molina con la implementación del «Programa de Vigilancia Sanitaria de Parques y Jardines». A partir del 2011 se realizó la refacción y mantenimiento de las veredas, caminos y senderos de los parques; en 2012 se hizo el mantenimiento y colocación de nuevos tachos de basura y en 2013 todos los parques públicos del distrito contaban con letreros de tenencia responsable y recojo de excretas. Así mismo, a partir de 2013-2014 se vio un aumento gradual y sustancial en el servicio de corte de césped y recolección de maleza $(>66 \%)$ y en el servicio de atención sanitaria $(>40 \%)$, basada principalmente en la promoción de campañas de desparasitación, charlas informativas de concientización hacia los dueños de las mascotas (tenencia responsable, recojo de excretas, etc.) y en el registro obligatorio de las mascotas en la municipalidad (Munimolina, 2016).

Dentro de los estudios más actuales, Cáceres et al. (2017) reportaron para la ciudad de Abancay, Perú, en 2012 la ausencia de parques amigables, $38.1 \%$ de parques poco amigables y $61.9 \%$ de parques no amigables, con prevalencias de Toxocara spp del 50 y $76.9 \%$, respectivamente, resultado que difiere en gran medida con los hallados en el presente estudio $(0.76 \%)$, sugiriendo la poca participación de la municipalidad de dicha ciudad en implementar las medidas dictadas por DIGESA mediante el «Programa de Vigilancia Sanitaria de Parques y Jardines».

\section{Conclusiones}

- La prevalencia de Toxocara spp en parques públicos del distrito de La Molina, Lima, fue $0.76 \%$.

- Es importante el compromiso y la activa participación de las municipalidades en la implementación del «Programa de Vigilancia Sanitaria de Parques y Jardines» para lograr cumplir con los objetivos propuestos en dicho programa.

\section{Literatura Citada}

1. Acha P, Szyfres B. 2003. Zoonoses and communicable diseases common to man and animals. Vol 3. Parasitoses. Washington DC, USA: Pan American Health Organization. $395 \mathrm{p}$.

2. Aguinaga J, Alva R, Livia G 2002. Prevalencia de huevos de Toxocara spp en parques y jardines del distrito de Ferreñafe. En: V Congreso Peruano de Parasitología. Trujillo. 
3. Alonso JM, Luna AC, Fernández GJ, Bojanich MV, Alonso ME. 2006. Huevos de Toxocara en suelos destinados a la recreación en una ciudad de Argentina. Acta Bioquim Clin L 40: 219-222.

4. Archelli S, Kozubsky L. 2008. Toxocara y Toxocariosis. Acta Bioquim Clin L 42: 379-384.

5. Armstrong WA, Oberg C, Orellana JJ. 2011. Presencia de huevos de parásitos con potencial zoonótico en parques y plazas públicas de la ciudad de Temuco, Región de La Araucanía, Chile. Arch Med Vet 43: 127-134.

6. Breña CJP, Hernández DR, Hernández PA, Castañeda IR, Espinoza BY, Roldán GW, Ramírez BC, et al. 2011. Toxocariosis humana en el Perú: aspectos epidemiológicos, clínicos y de laboratorio. Acta Med Peru 28: 228-236.

7. Cáceres P, Bustinza C, Valderrama P. 2017. Contaminación con huevos de Toxocara sp y evaluación sanitaria de parques en la ciudad de Abancay, Perú. Rev Inv Vet Perú 28: 376-386 doi: 10.15381/rivep.v28.i2.13064

8. Cajas J, Chávez A, Casas E. 2000. Prevalencia de huevos de Toxocara spp en parques públicos del cono sur de Lima Metropolitana. En: IV Congreso Peruano de Parasitología. Lima.

9. Canese A, Domínguez R, Otto C, Ocampos C, Mendonca E. 2003. Huevos infectivos de Toxocara en arenas de plazas y parques de Asunción, Paraguay. Rev Chil Pediatr 74: 611-616. doi: 10.4067/S0370-41062003000600010

10. Chávez AV, Casas EA, Serrano MM, Cajas JU, Velarde JO, La Rosa VV, López JT. 2002. Riesgo de contraer enfermedades parasitarias en los parques públicos de Lima y Callao. Rev Inv Vet Perú 13: 84-91. doi: 10.15381/ rivep.v11i1.6795.

11. Córdoba A, Ciarmela ML, Pezzani B, Gamboa MI, Marta De Luca M, Minvielle M, Basualdo JA. 2002. Presencia de parásitos intestinales en paseos públicos urbanos en La Plata, Argenti- na. Parasitol Latinoam 57: 25 - 29. doi: 10.4067/S0717-77122002000100007

12. Daniel W. 1996. Bioestadística base para el análisis de las ciencias de la salud. $3^{\circ}$ ed. México: Limusa. 875 p.

13. Dávalos AM, Pachas GO, Pérez EV. 2000. Toxocariosis en Canis familiaris y suelo en el distrito de Chincha Alta (1998-1999). En: IV Congreso Peruano de Parasitología. Lima.

14. [DIGESA] Dirección General de Salud Ambiental. 2009. Parques amigables y la salud pública. Bol Epidemiol 18: 416-417.

15. Goicochea A. 2012. Prevalencia de Toxocara canis en parques recreacionales del distrito de Trujillo durante el mes de julio. Tesis de Médico Veterinario. Trujillo: Univ. Alas Peruanas. 68 p.

16. Guerrero MO. 1975. Estudio de la contaminación de parques públicos de Lima Metropolitana con huevos de Toxocara spp. Tesis de Bachiller. Lima, Perú: Univ. Nacional Mayor de San Marcos. 12 p.

17. Huapaya HP, Espinoza Y, Roldán W, Jiménez S. 2009. Toxocariosis humana: ¿problema de Salud Pública? Anales Fac Med 70: 283-290.

18. La Rosa VV, Chávez VA, Casas AE. 2001. Contaminación de parques públicos del cono norte con huevos de Toxocara spp. Rev Inv Vet Perú 12: 116121. doi: 10.15381/rivep.v12i1.7431

19. López TF, Chávez VA, Casas AE. 2005. Contaminación de los parques públicos de los distritos de Lima Oeste con huevos de Toxocara sp. Rev Inv Vet Perú 16: 76-81. doi: 10.15381/ rivep.v16i1.1543

20. Montalvo SE, Villanueva BV, Marcelo $A E$, Mines HW, Cipriano FS, Peña VJ, et al. 2016. Soil contamination by Toxocara sp and other parasites of importance in public health of public parks of the Amarilis district (Huánuco, Peru). En: X Congreso Peruano de Parasitología. Lambayeque, Perú: Asociación de Parasitólogos del Perú (ASOPEPA). 
21. Munimolina. 2016. Municipalidad de La Molina. [Internet]. Disponible en: www.munimolina.gob.pe

22. Noé MN, Ulloa F, Peña P, Santos D, Fernández $C$, Anchante $H$, Terashima $A$, et al. 2011. Parasitosis zoonóticas en mascotas caninas y felinas de niños de educación primaria del cono norte de Lima, Perú. Rev Sapuvet Salud Pública 2: 15-24.

23. Omodu EA, Amuta EU, Unoqur LB, Okoye LA. 2003. Prevalence of Toxocara canis ova in dog faeces and soil samples collected from public parks in Makurdi. Nigerian J Parasitol 24: 137-142.

24. Romero NRC, García CAC, Mendoza $M G D$, Torres CNC, Ramírez DN. 2009. Contaminación por Toxocara spp en parques de Tulyehualco, México. Rev Cient 19: 253-256.

25. Serrano M, Chávez A, Casas E. 2000. Toxocariosis en parques del cono este de Lima. IV Congreso Peruano de Parasitología. Lima, Perú.
26. Schanstz, PM, Glickman LT. 1981. Epidemiology and pathogenesis of zoonotic toxocariasis. Epidemiol Rev 3: 230-250.

27. Tiyo R, Guedes TA, Falavigna DLM, Falavigna-Guilherme AL. 2008. Seasonal contamination of public squares and lawns by parasites with zoonotic potential in southern Brazil. J Helminthol 82: 1-6. Doi: 10.1017/S0022149X07870829

28. Velarde JA. 1999. Contaminación de los parques públicos de la Provincia Constitucional del Callao con huevos de Toxocara spp. Tesis de Médico Veterinario. Lima, Perú: Univ. Nacional Mayor de San Marcos. 62 p.

29. Zibaei M, Abdollahpour F, Birjandi M, Firoozeh F. 2010. Soil contamination with Toxocara spp eggs in the public parks from three areas of Khorram Abab, Iran. Nepal. Medical College J 12: 63-65. 